\title{
Design of A Dual-Wide Band BPF Utilizing Parallel Coupled Microstrip Lines and A Centered Arrow-Shaped Resonator
}

\author{
Ahmed Lateef Khudaraham ${ }^{1}$, Dhirgham Kamal Naji ${ }^{2 *}$
}

\section{Authors affiliations:}

1) Electronics and

Communications Eng. Dept.,

Al-Nahrain University,

Baghdad-Iraq.

ahmed.lateef771@gmial.com

$\left.2^{*}\right)$ Electronics and

Communications Eng. Dept.,

Al-Nahrain University,

Baghdad-Iraq.

dhrgham.kamal@gmail.com

\section{Paper History:}

Received: $27^{\text {th }}$ Jan. 2020

Revised: $12^{\text {th }}$ Feb. 2020

Accepted: $16^{\text {th }}$ March 2020

\begin{abstract}
This paper presents a dual wide-band band pass filter (DWB-BPF) by using two parallel, symmetrical micro-strip lines loaded by a centered resonator, consisting of a $\mathrm{T}$ - and a triangle-shaped geometry, attached at the lower and upper ends, respectively. The filter reveals good performance and both the passbands can be independently controlled by adjusting specific parts of the filter. The proposed BPF is simulated by using CST microwave studio package and the simulated result is verified experimentally with good agreement between the two results. The fabricated prototype BPF demonstrates two passbands located at $2.3 \mathrm{GHz}$ and $6.35 \mathrm{GHz}$ center frequencies with $39 \%$ and $23.6 \%$ of $3-\mathrm{dB}$ fractional bandwidth (FBW), respectively and a good insertion and return losses. The designed BPF can be targeted for wireless local area network (WLAN), WIFI and satellite communication systems.
\end{abstract}

Keywords: Bandpass Filter (BPF), Fractional Bandwidth (FBW), DualBand, Passband, WLAN.

$$
\begin{aligned}
& \text { تصميم مرشح حزم بكزمتين عريضتين باسستخدام خطوط شريطية دقيقة متوازية و } \\
& \text { مرنان في المركز على شكل السهم } \\
& \text { أحمد لطيف خدارحم ، ضرغام كمال ناجي }
\end{aligned}
$$

\section{Introduction}

Dual-band band pass filters (BPFs) are highly desirable nowadays, mainly those with wide-bands, good performance, good selectivity, low cost, and compact size. Due to the enormous progress in the $\mathrm{RF} /$ wireless communication systems, the radio spectrum has become progressively congested. The wide-band operation is required to allow users for achieving multiple services and not a single one. So, for these manners, the demand for dual-wide band operations has increased. Some of dual-band BPFs are, the dual-band filter in [1] was designed using steppedimpedance resonator (SIR) and T-shaped line. . The main step in this design was to change the quarter and half-wavelength micro-strip lines with a T-shaped line and two SIRs and the FBWs were $25 \%$ and $15.1 \%$, respectively. A novel dual-band BPF has been presented by using multi-mode resonator [2] with
FBW of $22.8 \%$ and $10.6 \%$. Another design of dualwide band BPF was proposed by using a T-shaped structure [3] and the FBW of both passbands were $28.8 \%$ and $22.7 \%$. The aforementioned three filters have a main drawback which was a very large size. In $[4,5]$ were developed a design for dual-band filters that quad-mode resonators were to provide more flexibility of controlling the bandwidths and the stopbands between the two passbands. A ring resonator with shorted stubs has been used to design a dual-band BPF with FBWs of $9.3 \%$ and $6.6 \%$ [6]. Moreover, the dualband BPFs with several transmission zeroes (TZs) were proposed by applying coupled steppedimpedance resonator (SIR) [7,8]. The design in [9] presents a dual-wide band filter with FBWs of $41.1 \%$ and $19 \%$ by using a planar structure and three sections of stubs that gave multiple TZs which were 
responsible for the good overall performance of the filter in terms of bandwidths and stopband.

Recently, a new quadruple-mode steppedimpedance square ring loaded resonator (SRLR) is anticipated, to design a high-performance dual-band differential BPF with FBWs of $10.4 \%$ and $3.6 \%$ respectively [10]. The filter in [11] also has utilized the SRLR to design a dual- narrow and wide-bandwidths BPF with FBWs of $8 \%$ and $39 \%$ and multi transmission zeros are produced near each passband edge, hence improving the selectivity of the passbands. A compact wide-band BPF is presented in [12] with wide bandwidths $(51.9 \%$ at $2.4 \mathrm{GHz}$ and $23.3 \%$ at 5.2 $\mathrm{GHz}$ ) by using the structure of open-/short-circuited stubs that provided the flexibility of manipulating the center frequencies by altering the impedance and the length of the stubs. However, although the previous reported BPF are successfully designed for operating at multiband, but most of them are either large in size or having complex structure.

Therefore, this paper presents a simple, compact, low cost, and effective design of dual-wide band BPF. Good performance has been achieved with high $\%$ FBW for each band. The stopband between the two passbands has been enhanced by the insertion of the triangle-shaped resonator. Design steps, results, and parametric studies are presented. Afterwards, the experimental results of the proposed BPF are illustrated.

\section{Design Concepts of the Proposed Dual-Band Filter}

In this section, the geometry of a compact dual wide-band band pass filter (DWB-BPF) is described and the three steps of the design approach applied to the designed filter is discussed in details. The concept of parallel coupled microstrip lines loaded by T-, L-, Fand arrow-shaped resonators is presented here to obtain DWB-BPF. Initially, the design approach starts with a conventional BPF, asymetrical coupled transmision lines loaded with T-saped strip structure, aiming to be resonated at $2.45 \mathrm{GHz}$. Then, intermediate two design steps are perfomed to achive BPF operated at a two different wide band centered at 2.45 and $6.5 \mathrm{GHz}$.

\subsection{Filter Geometry}

The final geometry of the proposed BPF is shown in Fig.1. As shown from Fig.1, the BPF is comprised of two coupled parallel lines and its lower end is folded and attached by an inverted-F and L-shaped stubs, and an inverted T-shaped stub with its upper end is loaded by the shape of an 'Arrow' geometry. The overall dimensions of the BPF $\left(L_{\text {sub }} \times W_{\text {sub }}\right)$ is $15 \mathrm{~mm} \times$ $25 \mathrm{~mm}$ and it is printed on the grounded FR4 substrate of thickness $h_{\text {sub }}$ of $1.6 \mathrm{~mm}$. A two $50 \Omega$ SMA connectors are used for transmission and reception of the signal through the two rectangular ports of dimensions $W_{1} \times L_{3}$. A two inverted Lshaped coupled transmission lines, vertical length $\left(L_{15}+L_{16}\right)$ and horizontal length $L_{17}$, are connected to the ports and the gap between them is $2 g+W_{3}$.

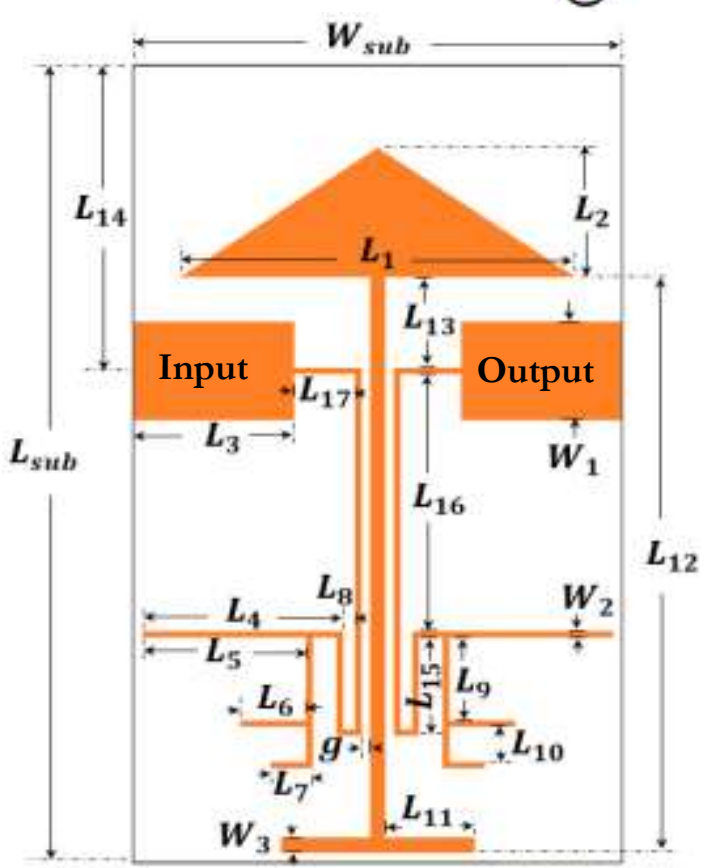

Figure (1): Full structure of the proposed DWBBPF.

Table (1): Optimized Geometric dimensions of the proposed DWB-BPF.

\begin{tabular}{|c|c|c|c|}
\hline Parameter & Value $\mathbf{m m}$ & Parameter & Value $\mathbf{m m}$ \\
\hline $\boldsymbol{L}_{\boldsymbol{s u b}}$ & 25 & $\boldsymbol{L}_{\mathbf{9}}$ & 2.6 \\
\hline $\boldsymbol{W}_{\boldsymbol{s u b}}$ & 15 & $\boldsymbol{L}_{\mathbf{1 0}}$ & 1 \\
\hline $\boldsymbol{h}_{\boldsymbol{s u b}}$ & 1.6 & $\boldsymbol{L}_{\mathbf{1 1}}$ & 2.75 \\
\hline $\boldsymbol{g}$ & 0.25 & $\boldsymbol{L}_{\mathbf{1}}$ & 18 \\
\hline $\boldsymbol{L}_{\mathbf{1}}$ & 12 & $\boldsymbol{L}_{\mathbf{1 3}}$ & 2.8 \\
\hline $\boldsymbol{L}_{\mathbf{2}}$ & 4 & $\boldsymbol{L}_{\mathbf{1 4}}$ & 9.5 \\
\hline $\boldsymbol{L}_{\mathbf{3}}$ & 5 & $\boldsymbol{L}_{\mathbf{1 5}}$ & 3 \\
\hline $\boldsymbol{L}_{\mathbf{4}}$ & 6.2 & $\boldsymbol{L}_{\mathbf{1 6}}$ & 8.1 \\
\hline $\boldsymbol{L}_{\mathbf{5}}$ & 5 & $\boldsymbol{L}_{\mathbf{1 7}}$ & 1.8 \\
\hline $\boldsymbol{L}_{\mathbf{6}}$ & 2 & $\boldsymbol{W}_{\mathbf{1}}$ & 3 \\
\hline $\boldsymbol{L}_{\mathbf{7}}$ & 1.2 & $\boldsymbol{W}_{\mathbf{2}}$ & 0.2 \\
\hline $\boldsymbol{L}_{\mathbf{8}}$ & 0.3 & $\boldsymbol{W}_{\mathbf{3}}$ & 0.5 \\
\hline
\end{tabular}

An inverted T-shaped strip of lengths $L_{11}$ and $L_{12}$ are placed at a gap distance $g$ between the two coupled parallel lines. A triangle-shaped structure of dimensions $L_{1} \times L_{2}$ is loaded at the upper end of Tshaped stub. The two inverted $\mathrm{F}$-shaped structures are characterized by the three geometric parameters $\left(L_{6}\right.$, $L_{7}, L_{9}$ and $\left.L_{10}\right)$ and the two L-shaped stubs are formed by the two dimensions $L_{4}$ and $L_{15}$ and placed at distance $L_{8}$ from the two coupled parallel lines. Table (1) lists the optimized dimensions of the proposed filter.

\subsection{Design Details}

Fig.2 demonstrates the procedure for the three design steps applied to develop the proposed DWBBPF, namely:

Step-1 (BPF 1): The construction of the single wideband BPF is represented in Fig.2 (a). The filter consists of two identical parallel micro-strip lines with length of around a quarter wavelength at the desired frequency, $2.45 \mathrm{GHz}$, and a third inverted T-shaped 
line is centered between these two parallel lines. This single wide-band BPF is printed on an FR-4 substrate with thickness of $1.6 \mathrm{~mm}$, dielectric constant 4.3 and tangent loss of 0.025 . The filter was simulated by using CST software package. The two port widths were selected to satisfy the $50 \Omega$ matching condition according to the transmission line theory. The response of the filter in terms of S-parameters is shown in Fig.3 (a). As noticed from Fig. 3 (a) that the filter reveals a wide-band operation centered at 2.65 $\mathrm{GHz}$ with wide stopband characteristic. The 3-dB FBW of the filter is $56 \%$ covering from $1.9 \mathrm{GHz}$ to 3.4 $\mathrm{GHz}$ with insertion loss of $-1 \mathrm{~dB}$ and a return loss of $-11 \mathrm{~dB}$ at the center frequency.

Step-2 (BPF 2): In this step, the previous filter BPF1 has been modified by folding the lower ends of the parallel micro-strip lines and extending them to the end sides of the substrate as shown in Fig.2 (b). This filter namely BPF2 provides a second wide-band with wide stopband between the two passbands and maintaining the first band but with a slight down shift to a lower center frequency. Filter response is illustrated in Fig.3 (b). The first passband was centered at $2.4 \mathrm{GHz}$ with a wide $3-\mathrm{dB}$ FBW of $48.7 \%$ $(1.83 \mathrm{GHz}-3.0 \mathrm{GHz})$ with $-0.64 \mathrm{~dB}$ of insertion loss and $-32 \mathrm{~dB}$ of return loss. The second passband operated at $6.59 \mathrm{GHz}$ center frequency with a $3-\mathrm{dB}$ FBW of $18 \%$ $(6.0 \mathrm{GHz}-7.18 \mathrm{GHz})$ with insertion and return losses of $-2.1 \mathrm{~dB}$ and $-18.2 \mathrm{~dB}$ at the center frequency. As observed, the second band is not sharp as needed, and the insertion loss is not as anticipated. Thus, it is required a modification on BPF 2 to covers the desired dual frequency bands.

Step-3 (BPF 3): In this third and final step of the procedure, proposed filter BPF3 is obtained by adding an inverted F- and L-shaped stubs to the folded sections of the parallel lines and a triangle shaped is loaded to the upper end of $\mathrm{T}$-shaped resonator structure as seen in Fig.2 (c) and its related response is illustrated in Fig.3 (c).

The operation and specifications of the designed DWB-BPF are summarized as:

a. The first passband is centered at $2.3 \mathrm{GHz}$ with a 3$\mathrm{dB}$ bandwidth of $814 \mathrm{MHz}(39 \% \mathrm{FBW})$ ranging from $1.86 \mathrm{GHz}$ to $2.70 \mathrm{GHz}$. The insertion and return losses of the first band were $-0.8 \mathrm{~dB}$ and $-23 \mathrm{~dB}$ respectively at the center frequency.

b. A good out-of-band rejection has been granted $(2.7 \mathrm{GHz}$ to $5.6 \mathrm{GHz})$ due to the generation of three transmission zeroes at $3.7 \mathrm{GHz}(-39 \mathrm{~dB}), 4.6 \mathrm{GHz}(-$ $36 \mathrm{~dB})$, and $4.8 \mathrm{GHz}(-42 \mathrm{~dB})$ resulting in sharper transition towards the second passband.

c. The second passband is centered at $6.35 \mathrm{GHz}$ with a 3-dB BW of $1500 \mathrm{MHz}$ (23.6\% FBW) oscillating from $5.6 \mathrm{GHz}$ to $7.1 \mathrm{GHz}$ with insertion and return losses of $-1.3 \mathrm{~dB}$ and $-21.2 \mathrm{~dB}$ respectively.

d. The greatest benefits of the proposed BPF were in its compactness, since its final dimensions is $25 \times 15$ $\mathrm{mm}^{2}$ adding to that simplicity of the design, and low cost.

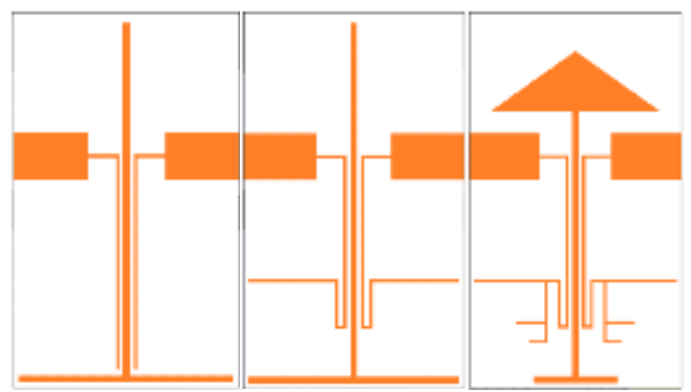

(a)

(b)

(c)

Figure (2): Geometry of various filters involved in the design evolution process.

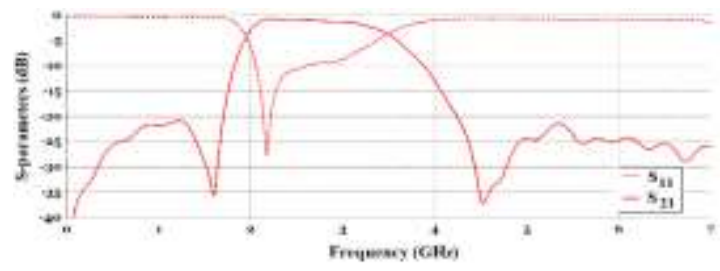

(a)

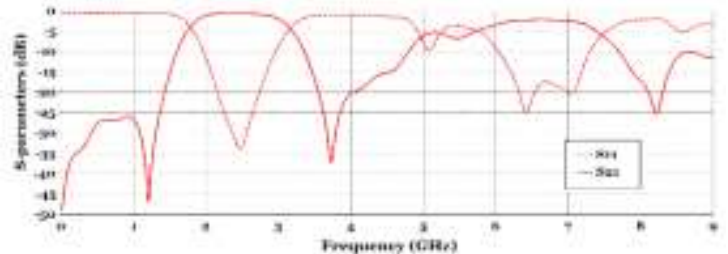

(b)

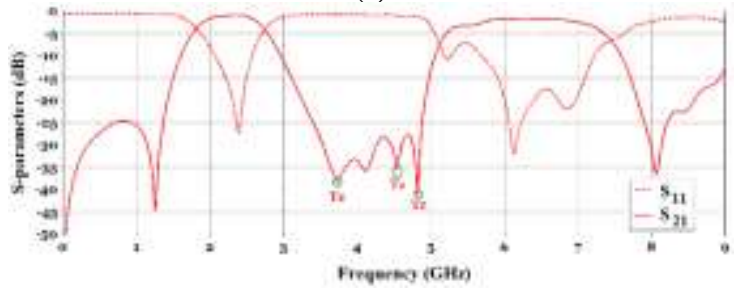

(c)

Figure (3): S-parameters of the three designed filters.

\section{Parametric Study}

The parametric study is essential to optimize filter performance and to observe the effect of each part on filter response. The filter performance is mainly effected by the width of the triangle $\left(L_{1}\right)$, length of the extended line to the sides of the substrate $\left(L_{4}\right)$, spacing between the two parallel lines and the folding part $(g)$.

\subsection{Effect of Varying $\boldsymbol{L}_{\mathbf{1}}$}

This parameter directly affects only the first passband and provides the benefit of controlling the first passband without effecting the second passband. The effect of alternating this parameter on filter response is revealed in Fig.4 (a).

\subsection{Effect of Varying $\boldsymbol{L}_{\mathbf{4}}$}

Conversely, the second passband also can be controlled independently by interchanging this parameter. Fig. 4(b) demonstrates the outcome 
resulting in changing of $L_{4}$ on S-parameters of the designed filter. As illustrated, increasing L2 will individually decrease the cutoff frequency of the second passband and maintain the first passband unchanged. For L2 $=5.5 \mathrm{~mm}$, the center frequency of the second passband has been shifted to $6.7 \mathrm{GHz}$ with $-1.7 \mathrm{~dB}$ insertion loss and $-26 \mathrm{~dB}$ return loss. For $\mathrm{L} 2=5.7 \mathrm{~mm}$, the center frequency of the second band was shifted back to $6.45 \mathrm{GHz}$ with insertion and return losses of $-1.8 \mathrm{~dB}$ and $-38 \mathrm{~dB}$ respectively. As a result, this parameter provides the independent control of the second passband without affecting the first one.

\subsection{Effect of Varying $L_{8}$}

This parameter also can individually change the second passband only without a major change regarding the first passband. This effect can be illustrated in Fig. 4(c).

For a better presentation for the effect of these three parameters, Table (2) shows the iteration of each parameter with their related effect on the center frequency $\left(f_{0}\right)$, insertion loss (IL) at $f_{0}$, and 3 -dB FBW.

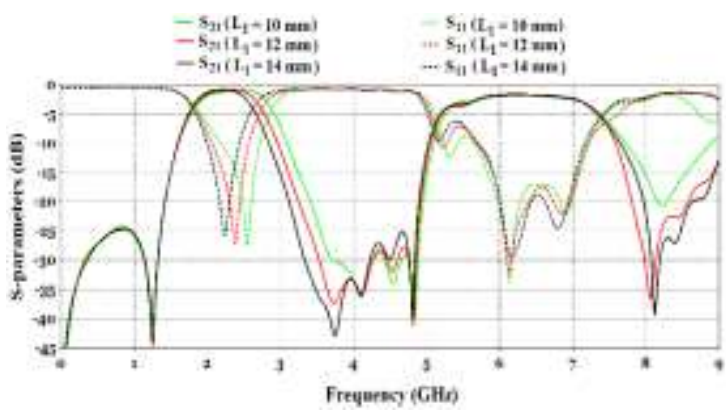

(a)

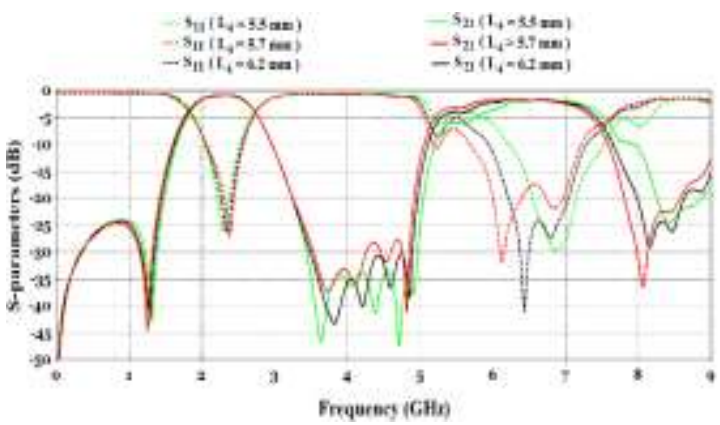

(b)

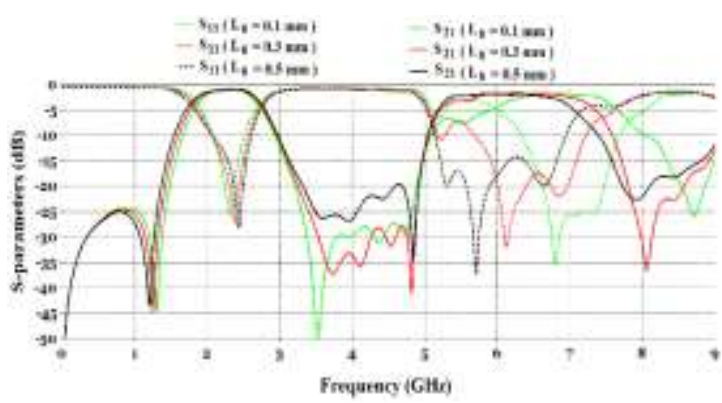

(c)

Figure (4): Effect of varying (a) L1, (b) L4 and (c) L8 on S-parameters of proposed filter.
Table (2): Parameters effect on filter response

\begin{tabular}{|c|c|c|c|c|c|c|c|}
\hline Parameter & $\begin{array}{l}\text { Value } \\
\text { (mm) }\end{array}$ & $\begin{array}{c}f_{o t} \\
\left(G H_{z}\right)\end{array}$ & $\begin{array}{c}f_{o z} \\
(G H z)\end{array}$ & $\begin{array}{l}L A \\
\text { (dB) }\end{array}$ & $\begin{array}{l}I L 2 \\
(d B)\end{array}$ & $\begin{array}{c}\frac{1}{F B W 1} \\
(\%)\end{array}$ & $\begin{array}{c}F B W^{2} \\
\text { (\%) }\end{array}$ \\
\hline \multirow{3}{*}{$L 1$} & 10 & 2.37 & 6.35 & 0.9 & 1.3 & 44 & 23.6 \\
\hline & 12 & 2.3 & 6.35 & 0.8 & 1.3 & 39 & 23.6 \\
\hline & 14 & 2.2 & 6.35 & 0.9 & 1.3 & 33 & 23.6 \\
\hline \multirow{3}{*}{$L 4$} & 5.5 & 2.28 & 6.7 & 0.8 & 1.6 & 37 & 18 \\
\hline & 5.7 & 2.3 & 6.48 & 0.8 & 1.6 & 39 & 24 \\
\hline & 6.2 & 2.3 & 6.35 & 0.8 & 1.3 & 39 & 23.6 \\
\hline \multirow{3}{*}{$L 8$} & 0.1 & 2.29 & 6.85 & 0.8 & 1.6 & 36 & 19 \\
\hline & 0.3 & 2.3 & 6.35 & 0.8 & 1.3 & 39 & 23.6 \\
\hline & 0.5 & 2.31 & 6 & 0.8 & 1.6 & 39 & 25.8 \\
\hline
\end{tabular}

\section{Surface Current Distribution}

The surface current distribution for the proposed $\mathrm{BPF}$ at frequencies of $2.3 \mathrm{GHz}, 4 \mathrm{GHz}, 6.35 \mathrm{GHz}$, and $8 \mathrm{GHz}$ are displayed in Fig. 5. The red color areas indicate the highest coupling, while the blue color areas indicate the lowest coupling. The maximum current density can be seen at $2.3 \mathrm{GHz}$ and $6.35 \mathrm{GHz}$ which are the passbands for the proposed BPF and this shows that the coupling at these two frequencies are the highest and the power can transfer from input to output port. For frequencies of $8 \mathrm{GHz}$ and $10 \mathrm{GHz}$ which are located at the stopbands, the lowest current density can be observed due to the weak coupling and the power is reflected back from output to input port.

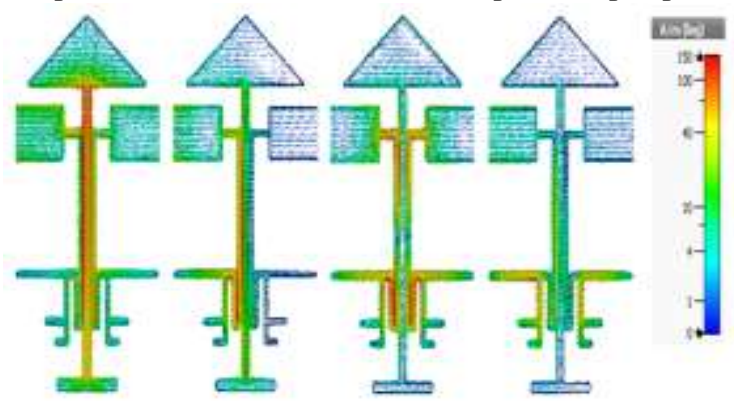

(a)

(b)

(c)

(d)

Figure (5): Surface current distribution: (a) 2.3GHz; (b) $4 \mathrm{GHz}$; (c) $6.35 \mathrm{GHz}$; (d) $8 \mathrm{GHz}$.

\section{Comparison with Reported BPFs}

The summary of the previously reported studies that have been published in recent years in comparison with the proposed BPF in terms of specifications is represented in Table (3).

Table (3): Comparison between recent reported dual-band BPFs and the proposed BPF.

\begin{tabular}{|c|c|c|c|c|}
\hline Reference & $\begin{array}{c}\boldsymbol{f}_{\boldsymbol{0}} \\
(\mathbf{G H z})\end{array}$ & $\begin{array}{c}\text { FBW } \\
(\boldsymbol{\%})\end{array}$ & $\begin{array}{c}\text { Size } \\
\left(\boldsymbol{\lambda}^{2} \boldsymbol{g}\right)\end{array}$ & Complexity \\
\hline$[\mathbf{1}]$ & $2.47 / 5.37$ & $25 / 5.1$ & $0.23 * 0.28$ & Low \\
\hline$[\mathbf{2}]$ & $2.5 / 5.3$ & $29.1 / 16.5$ & $0.58^{*} 0.22$ & Low \\
\hline$[\mathbf{3}]$ & $1.63 / 2.42$ & $28.8 / 22.7$ & $0.34 * 0.15$ & Medium \\
\hline$[\mathbf{6}]$ & $0.61 / 1.36$ & $32.3 / 10.5$ & NA & High \\
\hline$[\mathbf{8}]$ & $2.49 / 3.49$ & $15.6 / 8$ & $0.16 * 0.19$ & Low \\
\hline$[\mathbf{9}]$ & $0.705 / 1.37$ & $41.1 / 19$ & $0.3 *^{*} 0.05$ & Low \\
\hline
\end{tabular}




\begin{tabular}{|c|c|c|c|c|}
\hline$[10]$ & $2.6 / 5.8$ & $10.4 / 3.6$ & $0.26 * 0.34$ & Medium \\
\hline$[\mathbf{1 1}]$ & $2.4 / 4.0$ & $8.0 / 39$ & $0.48 * 0.09$ & Medium \\
\hline [12] & $2.4 / 5.2$ & $51.9 / 23.3$ & $0.28 * 0.20$ & Medium \\
\hline $\begin{array}{c}\text { This } \\
\text { work }\end{array}$ & $\mathbf{2 . 3 / 6 . 3 5}$ & $\mathbf{3 9 / 2 3 . 6}$ & $\mathbf{0 . 1 9 * 0 . 1 1}$ & Low \\
\hline
\end{tabular}

As can be seen from Table (3), wider bandwidth and more compactness have been achieved with the proposed BPF in comparison with previously reported BPFs.

\section{Measurement and Verification Results}

Fig. 6 shows the fabrication of the proposed BPF. The results of the CST simulation tool and the practical measurements are illustrated in Fig. 7. There is some mismatch, shifting and deviations due to the available commercial materials which caused the measurements errors.

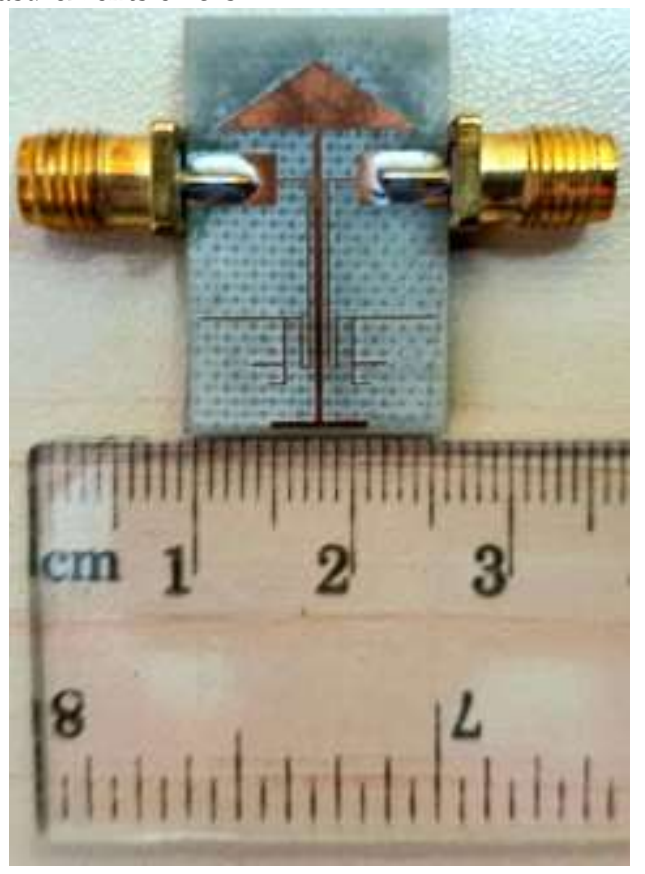

Figure (6): Fabrication of the proposed BPF.

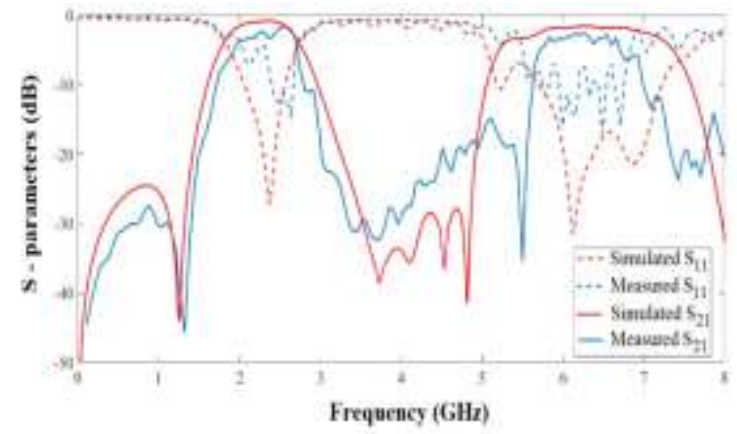

Figure (7): Simulation and measurement Sparameters of the proposed BPF.

\section{Conclusions}

In this paper, a compact and simple BPF design is proposed consisting of two folded parallel lines and a centered 'Arrow' shaped resonator. Two wide passbands are granted with three transmission zeros in the stopband between the two passbands. The proposed filter reveals the advantages of simplicity in the design, compactness, low cost since it is designed on a FR-4 substrate, good performance, and selectivity. This filter can be used in WiMAX (World Wide Interoperability for Microwave Access), WLAN (Wireless Local Area Network), and satellites applications. Also, the filter has been fabricated and good agreement has been observed between the simulation and measurement results.

\section{References:}

[1] Wenjie Feng; Quan Xue and Wenquan Che, "Compact dual-band bandpass filter based on stepped impedance resonators and T-shaped line", Microw. and Optic. Tech. Lett., Dec. 2010, Vol. 52, No. 4, pp. 2721-2724.

[2] W. J. Feng, W. Q. Che, and Q. Xue, "Novel dualband bandpass filter using multi-mode resonator", $4^{\text {th }}$ International High Speed Intelligent Communication Forum, May 2012.

[3] J.G. Zhou; W.J. Feng and W.Q. Che, "Dualwideband bandpass filter using $\mathrm{T}$-shaped structure based on transversal signal-interaction concepts", IEEE Elect. Lett., Nov. 2012, Vol. 48, No. 24, pp. 1539-1540.

[4] Shou-Jia Sun; Tao Su; Kun Deng; Bian Wu and Chang-Hong Liang, "Compact microstrip dualband bandpass filter using a novel stub-loaded quad-mode resonator", IEEE Microw. and Wirel. Compon. Lett., Sept. 2013, Vol. 23, No. 9, pp. 465467.

[5] Li Gao and Xiu Yin Zhang, "High-selectivity dualband bandpass filter using quad-mode resonator with source-load coupling", IEEE Microw. and Wirel. Compon. Lett., Sept. 2013, Vol. 23, No. 9, pp. 474-476.

[6] Runqi Zhang and Lei Zhu, "Design of a compact dual-band bandpass filter using coupled stepped impedance resonators", IEEE Microw. and Wirel. Compon. Lett., March 2014, Vol. 24, No. 3, pp. 155-157.

[7] Jin Shi; Longlong Lin; Jian-Xin Chen; Hui Chu and $\mathrm{Xu} \mathrm{Wu}$, "Dual-band bandpass filter with wide stopband using one stepped-impedance ring resonator with shorted stubs", IEEE Microw. and Wirel. Compon. Lett., July 2014, Vol. 24, No. 7, pp. 442-444.

[8] He Zhu and Amin M. Abbosh, "Single-and dualband bandpass filters using coupled steppedimpedance resonators with embedded coupledlines", IEEE Microw. and Wirel. Compon. Lett., Sept. 2016, Vol. 26, No. 9, pp. 675-677.

[9] Yongle Wu; Liwei Cui; Zheng Zhuang; Weimin Wang and Yuanan Liu, "A simple planar dual-band bandpass filter with multiple transmission poles and zeros", IEEE Trans. on Circ. and Syst., Jan. 2018, Vol. 65, No. 1, pp. 56-60. 
[10] Baoping Ren; Haiwen Liu; Zhewang Ma; et al. "Compact Dual-Band Differential Bandpass Filter Using Quadruple-Mode Stepped-Impedance Square Ring Loaded Resonator", IEEE Access, April 2018, Vol. 6, pp. 21850-21858.

[11] Min-Hang Weng; Siang-Wen Lan; et al. "Design of Dual-Band Bandpass Filter With Simultaneous Narrow- and Wide-Bandwidth and a Wide Stopband", IEEE Access, Oct. 2019, Vol. 7, pp. 147694-147703.

[12] Gen-Zhu Liang and Fu-Chang Chen, "A Compact Dual-Wideband Bandpass Filter Based on Open/Short-Circuited Stubs", IEEE Access, Jan. 2020, Vol. 8, pp. 20488-20492. 